\title{
NONLINEAR STABILITY OF A DIFFUSION EQUATION*
}

\author{
BY \\ GIOVANNI P. GALDI (Universita' di Napoli, Italy) \\ AND \\ ISOM H. HERRON ( Howard University)
}

1. Introduction. Recently [2], one of us has studied the linear stability of a nonlinear diffusion equation related to Burgers' equation

$$
u_{t}+u u_{y}=u_{x x}+u_{y y}, \quad-\infty<x, y<\infty, t>0,
$$

for the special solution

$$
U(y)=-2 \tanh y .
$$

There is no parameter in the problem, and it was shown that this solution is stable according to linear theory in the following way. With the assumption

$$
u=U(y)+\phi(y) e^{i \alpha(x-c t)}
$$

the solution is linearly stable for all $\alpha>0$, in that the only eigenvalue is $c=-i \alpha$. The main reason for the previous study was to examine the spectral problem which arises in trying to expand a disturbance in the eigenfunctions of the linearized stability equation. It was shown that in a weighted Hilbert space the spectrum is real and the usual spectral resolution by contour integration over the spectrum of the resolvent of the linearized operator is thereby possible. The space chosen was

$$
\mathscr{H}=\left\{\phi \mid \phi \cosh y \in \mathscr{L}_{2}(-\infty, \infty)\right\}
$$

with the inner product

$$
\langle\phi, \psi\rangle=\int_{-\infty}^{\infty} \phi(y) \bar{\psi}(y) \cosh ^{2} y d y
$$

for $\phi, \psi \in \mathscr{H}$.

* Received June 16, 1982. The first author worked under the auspices of GNFM of the Italian Research Council (C.N.R.) and the second author was partially supported through contracts with the U.S. Office of Naval Research (ONR), and the U.S. Army Research Office (ARO). 
There is still the question of the nonlinear stability of the equation, and the purpose here is to perform an energy analysis using weighted energy methods [1]. It is proved that disturbances which are either periodic in $x$, or have a Fourier transform in $x$, are stable according to energy theory for all sufficiently small initial values of the (weighted) energy. The case independent of $x$ has been ably handled elsewhere [4]. It was proved there that a type of orbital stability holds, but stability according to energy theory (asymptotic stability in the mean), is not to be expected. The present work goes further and delineates the stability boundary between linear and nonlinear stability. Further applications to exterior domain problems in mechanics are anticipated.

2. Energy Methods. A disturbance $v(x, y, t)$ to $u$ in (1.1) satisfies

$$
v_{t}+(U v)_{y}+v v_{y}=v_{x x}+v_{y y} .
$$

The weighted energy is defined as

$$
E(t)=\frac{1}{2} \int_{a} w v^{2} d a, \quad w>0,
$$

where $a=\{(x, y) \mid x \in X, y \in(-\infty, \infty)\}$. The set $X=(-\infty, \infty)$ when $v$ has a Fourier transform in $x$, or $X=[0,2 \pi / \alpha)$ if $v$ is periodic in $x$ with period $2 \pi / \alpha$.

The form of the weighted energy is suggested by the linearized analysis, but also occurs naturally when $w=\exp \left(-\int^{y} U(y) d y\right)=\cosh ^{2} y$ is introduced. Both sides of the perturbation equation (2.1) are multplied by $w v$ giving after integration by parts

$$
\frac{1}{2} \frac{d}{d t} \int_{a} w v^{2} d a=-\int_{a} w\left(v_{y}^{2}+v_{x}^{2}\right) d a-\int_{a} w U_{y} v^{2} d a-\int_{a} w v^{2} v_{y} d a .
$$

Thus (2.3) may be written

$$
\frac{d E}{d t}=-D+2 \int_{a} v^{2} d a-\int_{a} \cosh ^{2} y v^{2} v_{y} d a
$$

where $D=\int_{a} w|\nabla v|^{2} d a=\int_{a} \cosh ^{2} y\left(v_{x}^{2}+v_{y}^{2}\right) d a$.

Set

$$
\mathscr{H}^{\prime}=\left\{f \mid \cosh y f, \cosh y f_{x}, \cosh y f_{y} \in \mathscr{L}^{2}(a)\right\} .
$$

The following lemma holds.

LEMMA 1. For all $f \in \mathscr{H}^{\prime}$ we have the inequality

$$
\int_{a} \cosh ^{2} y f^{2} d a \leqslant \int_{a} \cosh ^{2} y\left(f_{x}^{2}+f_{y}^{2}\right) d a .
$$

Proof. Integration by parts shows

$$
\begin{aligned}
\int_{a} \cosh ^{2} y f^{2} d a & =-\int_{a} \sinh ^{2} y f^{2} d a-2 \int_{a} \sinh y \cosh y f f_{y} d a \\
& \leqslant \int_{a} \cosh ^{2} y\left(f_{x}^{2}+f_{y}^{2}\right) d a .
\end{aligned}
$$


COROLLARY. The following inequality also holds:

$$
\int_{a} f^{2} d a \leqslant \int_{a} \cosh ^{2} y\left(f_{x}^{2}+f_{y}^{2}\right) d a,
$$

for all $f \in \mathscr{H}^{\prime}$.

Another lemma will also be needed.

LeMma 2. For all $f \in \mathscr{H}^{\prime}$

$$
\left\{\int_{a}(\cosh y f)^{4} d a\right\}^{1 / 4} \leqslant c\left\{\int_{a} \cosh ^{2} y\left(f_{x}^{2}+f_{y}^{2}\right) d a\right\}^{1 / 2},
$$

where $c$ is a constant independent of $f$.

Proof. It is well known that for all $\psi$ such that $\psi, \psi_{x}, \psi_{y} \in \mathscr{L}^{2}(a)$, the following inequality holds [3]:

$$
\left\{\int_{a} \psi^{4} d a\right\}^{1 / 4} \leqslant c_{1}\left[\left\{\int_{a} \psi^{2} d a\right\}^{1 / 2}+\left\{\int_{a}\left(\psi_{x}^{2}+\psi_{y}^{2}\right) d a\right\}^{1 / 2}\right]
$$

where $c_{1}$ does not depend on $\psi$. Put $\psi=\cosh y f, f \in \mathscr{H}^{\prime}$, and consider the term $\psi_{y}^{2}$. It is

$$
\psi_{y}^{2}=\left(\sinh y f+\cosh y f_{y}\right)^{2} \leqslant 2 \cosh ^{2} y\left(f^{2}+f_{y}^{2}\right) .
$$

Therefore, the lemma follows from (2.8), (2.9) and Lemma 1.

Set

$$
\lambda=\max _{v \in \mathscr{H}^{\prime}} \frac{2 \int_{a} v^{2} d a}{\int_{a} \cosh ^{2} y\left(v_{x}^{2}+v_{y}^{2}\right) d a} .
$$

Thanks to the corollary to Lemma 1 , it follows that this maximum problem is well set. From (2.4) we obtain

$$
d E / d t \leqslant(\lambda-1) D+N
$$

where

$$
N=\frac{2}{3} \int_{a} \sinh y \cosh y v^{3} d a .
$$

From Lemma 2 and Schwarz's inequality it follows that

$$
|N| \leqslant \kappa E^{1 / 2} D \quad(\kappa>0, \text { const. }) .
$$

With (2.12), (2.11) implies

$$
d E / d t \leqslant\left(\lambda-1+\kappa E^{1 / 2}\right) D .
$$

Therefore, if $\lambda<1$, the linearized equation is certainly stable. Moreover, this allows us to choose $E(0)$ sufficiently small so that $E(t) \rightarrow 0$ as $t \rightarrow \infty$. For example, if

$$
0<E^{1 / 2}(0) \leqslant(1-\lambda) / 2 \kappa,
$$


$d E(0) / d t \leqslant((\lambda-1) / 2) D(0)<0$, so $E$ is initially decreasing. In fact, for every value of $t$ for which

$$
\begin{aligned}
\lambda-1+\kappa E^{1 / 2}(t) & \leqslant 0, \\
\frac{d E}{d t} & \leqslant 2\left(\lambda-1+\kappa E^{1 / 2}\right) E \leqslant 0,
\end{aligned}
$$

by virtue of (2.13) and (2.6). Thus, since $E(t)$ is initially decreasing, (2.16) holds on some interval $\left[0, t_{0}\right], t_{0}>0$, implying that $E\left(t_{0}\right) \leqslant E(0)$. Thus $d E\left(t_{0}\right) / d t<0$, and (2.16) may be continued beyond $t_{0}$. Repetition of the preceeding argument gives

$$
E^{1 / 2}(t) \leqslant(1-\lambda) / 2 \kappa, \quad \forall t>0 .
$$

Substituting (2.17) into (2.13) and employing (2.6) it follows that

$$
d E / d t \leqslant-(1-\lambda) E \text {. }
$$

Therefore

$$
E(t) \leqslant E(0) e^{-(1-\lambda) t},
$$

whenever $\lambda<1$ and (2.14) is satisfied. So, if $\lambda<1$ we have proved the following theorem: THEOREM. Let the weighted energy of the perturbation to (1.1), (1.2) be given by $E(t)$ in (2.2). Then if $E(0)$ is sufficiently small, $E(t) \rightarrow 0$ as $t \rightarrow \infty$. That is, $U(y)$ is conditionally asymptotically stable in the mean.

The proof that $\lambda<1$ is given in the next section.

3. The linear / nonlinear stability boundary. The next step is to investigate the conditions under which $\lambda<1$. First notice that the Euler-Lagrange equation associated with the maximum problem (2.10) is

$$
\cosh ^{2} y \Phi_{x x}+\left(\cosh ^{2} y \phi_{y}\right)_{y}+2 \Phi / \lambda=0 .
$$

where $\Phi \in \mathscr{H}^{\prime}$. Solutions of (3.1) are sought such that when $X=(-\infty, \infty)$, $\Phi$ has Fourier transform in $x$ given by

$$
\phi(y ; \alpha)=\int_{-\infty}^{\infty} \Phi(x, y) e^{i \alpha x} d x,
$$

or when $X=[0,2 \pi / \alpha), \Phi$ has a finite Fourier transform in $x$ given by

$$
\phi(y ; \alpha)=\int_{0}^{2 \pi / \alpha} \Phi(x, y) e^{i \alpha x} d x .
$$

The resulting equation for $\phi(y)$ in either case is

$$
-\phi^{\prime \prime}+(U \phi)^{\prime}+\alpha^{2} \phi=\mu\left(\operatorname{sech}^{2} y\right) \phi
$$

with

$$
\mu=2\left(\lambda^{-1}-1\right) .
$$

Solutions of (3.3a) are sought for which $\phi \in \mathscr{H},(1.4)$. For that reason, and because the equation is not in symmetric form set $\psi=\cosh y \phi$. When $\phi \in \mathscr{H}, \psi \in \mathscr{L}_{2}(-\infty, \infty)$, and $\psi$ satisfies the symmetric equation

$$
-\psi^{\prime \prime}+\left(\alpha^{2}+1\right) \psi=(\mu+2) \operatorname{sech}^{2} y \psi .
$$


Thus, it is clear that all admissible eigenvalues $\mu$ are real, though this is to be expected since (3.3a) came from a variational problem. Moreover, (3.4) has only eigenvalues, i.e. its continuous spectrum is void, because of the presence of the "inverse weight" $\rho(y)=$ $1 / w(y)=\operatorname{sech}^{2} y$ [6]. Furthermore, it is obvious that $\mu+2>0$, but it is also true, but not so obvious that $\mu>0$. This latter condition means with (3.3b), that $\lambda<1$. With our expectations clearly in view we return to the non-symmetric equation (3.3a).

Consider equation (3.3a) at criticality, namely when $\mu=0$ :

$$
-\phi^{\prime \prime}+\alpha_{0}^{2} \phi+(U \phi)^{\prime}=0 .
$$

It is noteworthy that equation (3.5) coincides with the one obtained from the linear theory at criticality [2]. This is due to the fact that the linear operator involved is symmetrizable. From the analysis developed in [2], it follows that (3.5) has a solution in the space $\mathscr{H}$, if and only if $\alpha_{0}=0$.

LEMMA 3. The eigenvalues $\mu$ of (3.3a) satisfy the estimate

$$
\alpha^{2} \leqslant \mu \text {. }
$$

Proof. To show (3.6), a standard argument is employed. Let $\phi$ be a solution of (3.3a) corresponding to $\alpha$ and $\mu(\alpha)$. This solution depends continuously on these parameters. Consider two such solutions and label them with subscripts. It follows that

$$
\begin{aligned}
& -\phi_{1}^{\prime \prime}+\alpha_{1}^{2} \phi_{1}+\left(U \phi_{1}\right)^{\prime}=\mu_{1} \operatorname{sech}^{2} y \phi_{1}, \\
& -\phi_{2}^{\prime \prime}+\alpha_{2}^{2} \phi_{2}+\left(U \phi_{2}\right)^{\prime}=\mu_{2} \operatorname{sech}^{2} y \phi_{2} .
\end{aligned}
$$

Multiply (3.7b) by $\cosh ^{2} y \phi_{2}$ and (3.7a) by $\cosh ^{2} y \phi_{1}$, integrating by parts over $(-\infty, \infty)$ and subtracting the relations so obtained to deduce that

$$
\left(\mu_{1}-\mu_{2}\right) \int_{-\infty}^{\infty} \phi_{1} \phi_{2} d y=\left(\alpha_{1}^{2}-\alpha_{2}^{2}\right) \int_{-\infty}^{\infty} \cosh ^{2} y \phi_{1} \phi_{2} d y .
$$

Allowing the solutions to coalesce we have

$$
\frac{d}{d \mu}\left(\alpha^{2}\right)=\frac{\int_{-\infty}^{\infty} \phi^{2} d y}{\int_{-\infty}^{\infty}(\cosh y \phi)^{2} d y} \leqslant 1
$$

for all $\phi \in \mathscr{H}$. Therefore,

$$
\alpha^{2}(\mu)-\alpha^{2}(0) \leqslant \mu .
$$

On the other hand from (3.5), $\alpha^{2}(0)=0$ and inequality (3.6) is proved.

Thus the inequality (3.6) holds, as in the linear theory. It also follows that $\lambda<1$, $\forall \alpha \neq 0$, thus the linear and nonlinear stability limits coincide [1]. For the linearized stability equation, the eigenvalue with smallest real part has a positive real part and the (symmetrizable) linear operator arising from the variational equation has a positive smallest eigenvalue. Put more sufficiently, linear stability implies nonlinear stability and vice versa.

4. Conclusions and implications. The stability of the solution (1.2) to equation (1.1) has been established for both infinitesimal and finite disturbances of sufficiently small initial 
energy. The energy criterion used is a weighted energy whose weight function symmetrizes the linearized operator. It is expected that this solution can be proved to be stable by other criteria such a weighted norms in Banach spaces [5]. The particular choice here is motivated by the physical implications of using the weighted $\mathscr{L}_{2}$ norm.

It is known that in the case of boundary layer flows, which motivated this investigation, the linearized operator is not symmetrizable. However its spectrum takes a fairly simple form, consisting of a finite number of eigenvalues and a continuous spectrum lying along a Jordan curve. The ability to "confine" the spectrum of the linearized operator has been used in other studies [5]. But any application to the nonlinear stbility stability of boundary layer solutions to the Navier-Stokes equations still lies before us.

\section{REFERENCES}

[1] G. P. Galdi and B. Straughan, Exchange of stability, symmetry and nonlinear stability, Arch. Rat. Mech. Anal. (to appear).

[2] I. H. Herron, A diffusion equation illustrating spectral theory for boundary layer stability, Studies in Appl. Math. 67, 231-241 (1982)

[3] O. A. Ladyzhenskaya, The mathematical theory of viscous incompressible flow, Gordon and Breach, New York. 1963

[4] L. A. Peletier, Asymptotic stability of travelling waves, in Instability of Continuous Systems (H. Leipholz, ed.), Springer-Verlag, Berlin, 1970, 418-422

[5] D. H. Sattinger, Weighted norms for the stability of travelling waves, J. Diff. Eqns. 25, 130-144 (1977)

[6] E. C. Titchmarsh, Eigenfunction expansions associated with second-order differential equations, University Press, Oxford, 1946 